\title{
Smoothed Analysis of Multi-Item Auctions with Correlated Values $^{*}$
}

\author{
ALEXANDROS PSOMAS, Carnegie Mellon University, USA \\ ARIEL SCHVARTZMAN, Princeton University, USA \\ S. MATTHEW WEINBERG ${ }^{\dagger}$, Princeton University, USA
}

Consider a seller with $m$ heterogeneous items for sale to a single additive buyer whose values for the items are arbitrarily correlated. It was previously shown that, in such settings, distributions exist for which the seller's optimal revenue is infinite, but the best "simple" mechanism achieves revenue at most one (Briest et al [2], Hart and Nisan [6]), even when $m=2$. This result has long served as a cautionary tale discouraging the study of multi-item auctions without some notion of "independent items".

In this work we initiate a smoothed analysis of such multi-item auction settings. We consider a buyer whose item values are drawn from an arbitrarily correlated multi-dimensional distribution then randomly perturbed with magnitude $\delta$ under several natural perturbation models. On one hand, we prove that the [2,6] construction is surprisingly robust to certain natural perturbations of this form, and the infinite gap remains On the other hand, we provide a smoothed model such that the approximation guarantee of simple mechanisms is smoothed-finite. We show that when the perturbation has magnitude $\delta$, pricing only the grand bundle guarantees an $O(1 / \delta)$-approximation to the optimal revenue. That is, no matter the (worst-case) initially correlated distribution, these tiny perturbations suffice to bring the gap down from infinite to finite. We further show that the same guarantees hold when $n$ buyers have values drawn from an arbitrarily correlated $m n$-dimensional distribution (without any dependence on $n$ ). Taken together, these analyses further pin down key properties of correlated distributions that result in large gaps between simplicity and optimality.

CCS Concepts: • Theory of computation $\rightarrow$ Algorithmic mechanism design; Computational pricing and auctions.

Additional Key Words and Phrases: approximate mechanism design, multi-item mechanism design, correlated distributions, smoothed analysis

\section{ACM Reference Format:}

Alexandros Psomas, Ariel Schvartzman, and S. Matthew Weinberg. 2019. Smoothed Analysis of Multi-Item Auctions with Correlated Values. In ACM EC '19: ACM Conference on Economics and Computation (EC '19), fune 24-28, 2019, Phoenix, AZ, USA. ACM, New York, NY, USA, 2 pages. https://doi.org/10.1145/3328526.3329563

How should a revenue-maximizing seller sell heterogeneous goods to interested buyers? This problem has been extensively studied by economists and computer scientists alike, from a variety of perspectives. One major highlight from these works is numerous impossibility results, essentially proving that one cannot hope to find a mechanism that is simple, yet optimal in general settings [2,6]. A major highlight on the flip side are numerous approximation results, where simple mechanisms are now known to be approximately optimal in quite general settings $[1,3-5,7]$.

\footnotetext{
*The full version of this paper can be found in [8].

† Supported by NSF CCF-1717899. EC '19, June 24-28, 2019, Phoenix, AZ, USA

(C) 2019 Copyright held by the owner/author(s).

ACM ISBN 978-1-4503-6792-9/19/06.

https://doi.org/10.1145/3328526.3329563
}

Permission to make digital or hard copies of part or all of this work for personal or classroom use is granted without fee
provided that copies are not made or distributed for profit or commercial advantage and that copies bear this notice and
the full citation on the first page. Copyrights for third-party components of this work must be honored. For all other uses,
contact the owner/author(s). 
Even as these approximation results generalized in various directions, they relied on the assumption that the values for the items are drawn independently. While "independent items" is a perfectly natural assumption (and we have greatly deepened our understanding of mechanism design under it), it was never intended to be ubiquitous in all future works. This is especially true due to the nature of the motivating impossibility result: the $\mathcal{D}$ witnessing these impossibilities is so carefully crafted that it is far removed from any "real-world" motivation. The thoughtful reader may at this point be thinking: if this construction is so fragile that even a tiny deviation breaks it, perhaps a smoothed analysis might prove insightful. Indeed, this is the focus of this paper.

Given an arbitrarily correlated distribution $\mathcal{D}$, its smoothing $\hat{\mathcal{D}}$ first draws a valuation vector $\mathbf{v}$ from $\mathcal{D}$, and then randomly perturbs $\mathbf{v}$ to $\hat{\mathbf{v}}$. We consider two natural models. In the first model, which we call Rectangle-Shift, values $(v, w)$ are perturbed to $\left(v+\varepsilon_{1} v, w+\varepsilon_{2} w\right)$, where each $\varepsilon_{i}$ is drawn independently from $U[0, \delta]$. Our first main result proves that in fact the infinite gap persists in this model! This indeed provides insight in a natural smoothed model and helps explain more clearly where the infinite gap comes from in the constructions of [2,6].

Theorem 1. For all $\delta<1 / 2$, there exists a bivariate distribution $\mathcal{D}$ such that for its corresponding perturbed distribution $\hat{\mathcal{D}}$ in the Rectangle-Shift model, $\operatorname{BREV}(\hat{\mathcal{D}}) \in O(1)$ and $\operatorname{REV}(\hat{\mathcal{D}})=\infty$.

In the second model, which we call Square-Shift, values $(v, w)$ are perturbed to $\left(v+\varepsilon_{1} \max \{v, w\}, w+\right.$ $\left.\varepsilon_{2} \max \{v, w\}\right)$, where each $\varepsilon_{i}$ is drawn independently from $U[0, \delta]$. Our second main result shows that $\operatorname{BREv}(\hat{\mathcal{D}}) \in \Omega(\delta \cdot \operatorname{Rev}(\hat{\mathcal{D}}))$.

Theorem 2. In the Square-Shift model, for a single additive buyer and 2 items

$$
\operatorname{REV}(\hat{\mathcal{D}}) \leq \frac{\sqrt{2} \pi(1+\delta)^{3} \log (1+\delta)}{\delta^{2}} \operatorname{BREV}(\hat{\mathcal{D}}) \in O\left(\frac{1}{\delta} \cdot \operatorname{BREV}(\hat{\mathcal{D}})\right)
$$

Our results for this model extend to multi-item, multi-bidder settings and settings beyond those where the buyers' valuation functions are additive across the items. It is worth noting that the approximation factor depends exponentially on the number of items (and this is nearly tight), but does not depend on the number of (possibly correlated) bidders.

\section{REFERENCES}

[1] Moshe Babaioff, Nicole Immorlica, Brendan Lucier, and S Matthew Weinberg. 2014. A simple and approximately optimal mechanism for an additive buyer. In 2014 IEEE 55th Annual Symposium on Foundations of Computer Science. IEEE, 21-30.

[2] Patrick Briest, Shuchi Chawla, Robert Kleinberg, and S Matthew Weinberg. 2015. Pricing lotteries. Fournal of Economic Theory 156 (2015), 144-174.

[3] Shuchi Chawla, Jason D. Hartline, and Robert Kleinberg. 2007. Algorithmic Pricing via Virtual Valuations. In Proceedings of the 8th ACM Conference on Electronic Commerce (EC '07). ACM, New York, NY, USA, 243-251. https://doi.org/10. $1145 / 1250910.1250946$

[4] Shuchi Chawla, Jason D Hartline, David L Malec, and Balasubramanian Sivan. 2010. Multi-parameter mechanism design and sequential posted pricing. In Proceedings of the forty-second ACM symposium on Theory of computing. ACM, 311-320.

[5] Sergiu Hart and Noam Nisan. 2012. Approximate revenue maximization with multiple items. In Proceedings of the 13th ACM Conference on Electronic Commerce. ACM, 656-656.

[6] Sergiu Hart and Noam Nisan. 2013. The menu-size complexity of auctions. arXiv preprint arXiv:1304.6116 (2013).

[7] Jason D. Hartline and Tim Roughgarden. 2009. Simple Versus Optimal Mechanisms. In Proceedings of the 10th ACM Conference on Electronic Commerce (EC '09). ACM, New York, NY, USA, 225-234. https://doi.org/10.1145/1566374.1566407

[8] Christos-Alexandros Psomas, Ariel Schvartzman, and S. Matthew Weinberg. 2018. Smoothed Analysis of Multi-Item Auctions with Correlated Values. CoRR abs/1811.12459 (2018). arXiv:1811.12459 http://arxiv.org/abs/1811.12459 